\title{
Aortic valve avulsion after proximal aortic dissection in the Marfan syndrome: echocardiographic features and management
}

\author{
J COLIN FORFAR, DAVID P DE BONO, KENNETH G REID \\ From the Departments of Cardiology and Cardiothoracic Surgery, Royal Infirmary, Edinburgh
}

The cardiovascular complications of the Marfan syndrome are principally responsible for the reduced life expectancy of patients with this disorder. ${ }^{1}$ Aneurysmal dilatation of the aortic root leads to progressive aortic regurgitation and predisposes to aortic rupture. Dissection of the proximal aorta and aneurysmal tears without dissection are also recognised. ${ }^{2}$ Recent surgical approaches to aortic root aneurysms in this syndrome have recommended replacement of the ascending aorta and aortic valve with coronary reimplantation..$^{3-5}$ Conservation of the aortic valve would offer several long-term advantages.

\section{Case report}

A 41-year-old woman, previously fit, collapsed at home after complaining of chest and back pain. On examination in hospital she was confused, pale, and hypotensive (blood pressure $90 / 60 \mathrm{~mm} \mathrm{Hg}$ in both arms), with intact normal volume peripheral pulses. Grade 3/6 aortic systolic and diastolic murmurs were audible. A chest radiograph showed cardiomegaly (cardiothoracic ratio 60\%), scoliosis, and prominence of the ascending aorta. The electrocardiogram showed lateral $\mathrm{T}$ wave inversion. She had arachnodactyly and a high arched palate but no other features of the Marfan syndrome.

Six hours later she deteriorated abruptly with reduction in both carotid pulse volumes. The systolic blood pressure in the right arm decreased to $50 \mathrm{~mm} \mathrm{Hg}$, the left arm blood pressure being $90 / 60 \mathrm{~mm} \mathrm{Hg}$, of low volume and with rapid upstroke. A gallop rhythm was audible, with no systolic murmur but a short staccato diastolic murmur grade $2 / 6$ was audible at the left sternal edge.

M-mode echocardiography showed a dilated hypercontractile left ventricle (EDD $6.7 \mathrm{~cm}$ ) with fluttering and premature closure of the mitral valve. The aortic valve echo appearances were unremarkable apart from early systolic closure of the anterior cusp, but a diastolic echo was visualised posterior to the valve, apparently within the left atrium. Two-dimensional echocardiography (Kontron Irex Mark III) in the long axis of the left ventricle showed flail aortic valve leaflets prolapsing in diastole into the left ventricular cavity (fig 1a). Aortic root dimensions were normal $(2.9 \mathrm{~cm})$ but the aorta was dilated distally. Aortography confirmed the root tear with dissection affecting the innominate and left carotid arteries and extending into the descending aorta. The ascending aorta was considerably dilated with free aortic regurgitation (fig $1 b$ ).

Address for reprint requests: Mr KG Reid, Department of Cardiothoracic Surgery, Royal Infirmary, Edinburgh, EH3 9YW
Cardiopulmonary bypass was established with the patient in a critical condition. A large fusiform aneurysm of the ascending aorta was visualised with aortic integrity maintained by a thin layer of adventitia. A tear affected $80 \%$ of the aortic circumference immediately above the valve. The dissection extended proximally and distally, allowing free prolapse of the intima and media into the left ventricular outflow tract. The aortic root was not dilated and the valve cusps appeared normal. After coronary perfusion the aortic wall supporting the valve commissures was dissected with preservation of the upper margin of the coronary ostia, and the attachments of the valve commissures were secured firmly to the adventitia. A Dacron graft was sculptured to the trimmed aortic root and sewn into place. The graft acted as an outer support for the aortic commissures. Distally the graft was cut obliquely to match the trimmed edge of the aorta and anastomosed just below the innominate artery (fig 2).

Bypass was discontinued uneventfully and the patient's subsequent progress was unremarkable, with no evidence of pulse or neurological deficit. Clinical cardiovascular examination at discharge four weeks later showed normal heart sounds with no murmurs. The blood pressure was $120 / 70 \mathrm{~mm} \mathrm{Hg}$ in both arms.

Aortic and mitral valve appearances were normal on repeat echocardiography before discharge, with a small reduction in ventricular dilatation (EDD $5.4 \mathrm{~cm}$ ). Microscopic examination of the aorta confirmed extensive disruption of the media with fragmentation of medial elastic fibres, in keeping with the Marfan syndrome. The patient has remained symptom free over a nine-month follow-up.

\section{Discussion}

Aortic histology in the Marfan syndrome typically shows fragmentation and degeneration of elastic and helical collagen fibres with defective biosynthesis and cross-linkage of collagen subchains. ${ }^{6}$ In the presence of annuloaortic ectasia and chronic regurgitation, surgical repair requires replacement of both the aortic valve and the ascending aorta. $^{34}$

In our patient, however, the aortic root dimensions were normal with a type I dissection ${ }^{7}$ and avulsion of the aortic valve. Necropsy studies ${ }^{8}$ suggest that this may be a relatively distinct lesion in the Marfan syndrome. Valve replacement may not be required, provided that the commissures can be resuspended against the aortic prosthesis. The surgical technique described offers the long-term advantages inherent in a native valve, thus avoiding problems related to valve degeneration, thrombosis, and haemorrhage. 


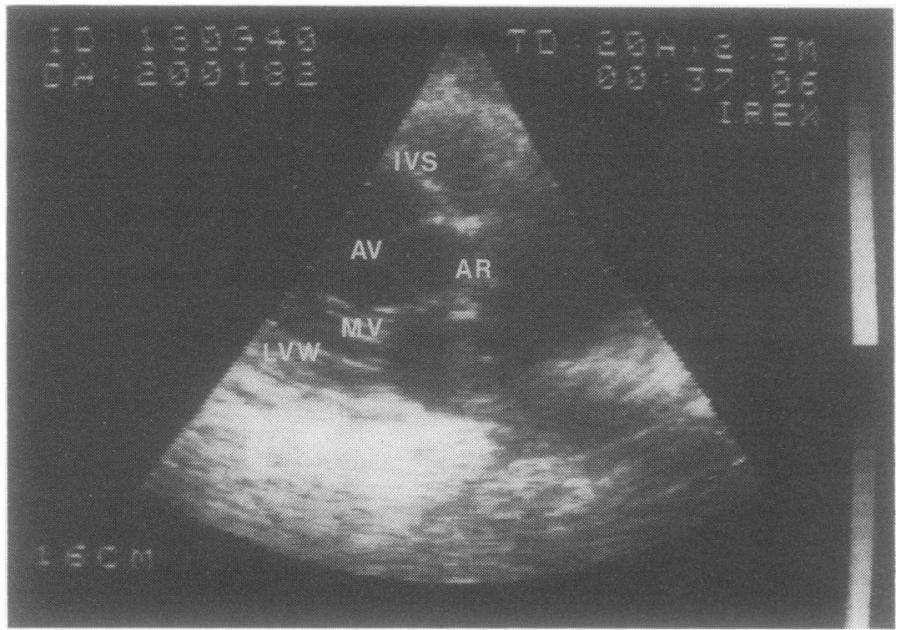

Fig 1 Echocardiography and angiographic appearances of aortic dissection and aortic valve avulsion. (a) Two-dimensional scan in diastole: the aortic valve and dissected intima prolapsed into the left ventricle (aortic root dimensions normal). (b) Fusiform aneurysm of the ascending aorta with free aortic regurgitation shown by aortography. The intimal flap in the ascending aorta extended into the innominate and left carotid arteries (arrows). Both subclavian vessels opacified poorly. $A R$-aortic root; $A V$-aortic valve; $I V S$-interventricular septum; $M V \longrightarrow$ mitral valve; $L V$-left ventricle; $L V W$-left ventricular free wall.

Fig 1a

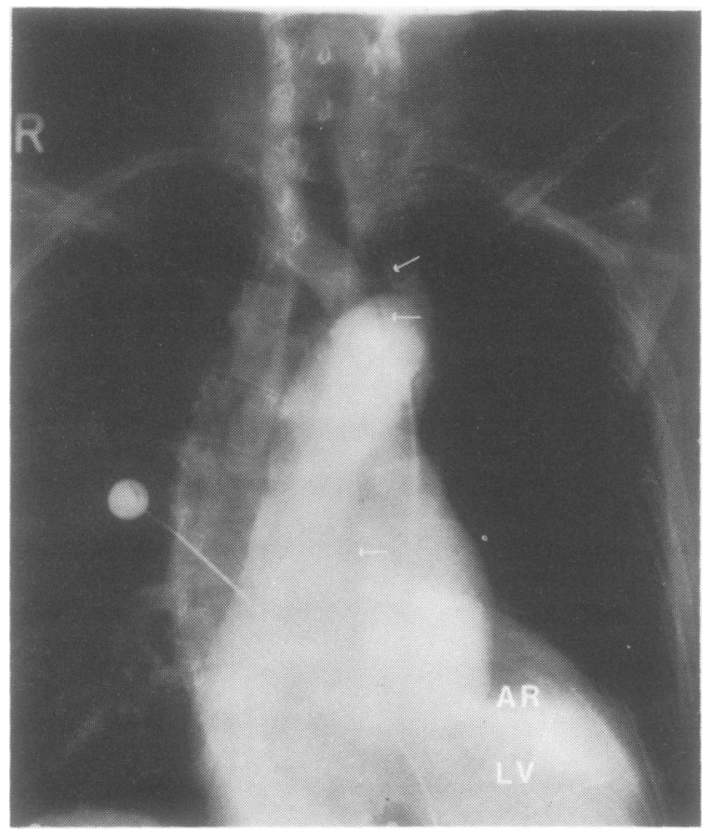

Fig 1b

Two-dimensional echocardiography accurately defined the aortic root dimensions and the gross valve and intimal prolapse in this patient. Single-element echocardiography had limitations because of difficulties in defining the aortic root in the presence of a flail valve. Other recognised features of aortic root dissection, including root image enlargement $^{9}$ and aortic cusp duplication, ${ }^{10}$ were not identified. In retrospect, aortography added little to the diagnosis and delayed surgical intervention in a critically ill patient.

Unfortunately, we have no means of identifying patients at high risk from acute aortic dissection in the Marfan syn-

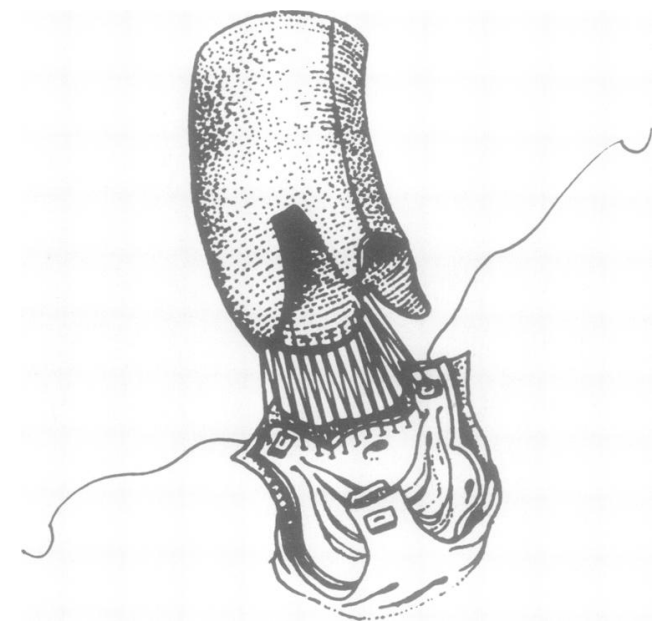

Fig 2 Replacement of the ascending aorta with conservation of the aortic valve. The upper margins of the attachments of the valve commissures were secured to the adventitial layer before they were approximated to a graft preshaped to match the trimmed aortic root. The upper end of the graft was trimmed to the aorta and anastomosed proximal to the innominate artery.

drome, especially as dissection may be more common in the absence of aortic root dilatation. ${ }^{8}$ Echocardiography, however, allows immediate diagnosis and early surgery without further investigation and may offer the best chance of a satisfactory outcome.

\section{References}

${ }^{1}$ Murdoch JL, Walker BA, Halpern BL, Kuzma JW, McKusick VA. Life expectancy and causes of death in the Marfan syndrome. N Engl J Med 1972;286:804-8. 
${ }^{2}$ Roberts WC. Aortic dissection: anatomy, consequences and causes. Am Heart J 1981;101:195-214.

${ }^{3}$ McDonald GR, Schaff HV, Pyeritz TE, McKusick VA, Gott L. Surgical management of patients with the Marfan syndrome and dilatation of the ascending aorta. J Thorac Cardiovasc Surg 1981;81:180-6.

${ }^{4}$ Bentall H, de Bono A. A technique for complete replacement of the ascending aorta. Thorax 1968;23:338-9.

${ }^{5}$ Donaldson RM, Emanuel RW, Olsen EGJ, Ross DN. Management of cardiovascular complications in Marfan syndrome. Lancet 1980;ii:1178-80.

' Scheck M, Siegel RC, Parker J, Chang YH, Fu JC. Aortic aneurysm in Marfan's syndrome: changes in the ultrastructure and composition of collagen.J Anat 1979;129:645-57.
' DeBakey MF, Henly WS, Cooley DA, Morris GC jun, Crawford ES, Beal AC. Surgical management of dissecting aneurysms of the aorta. J Thorac Cardiovasc Surg 1965;40:130-48.

${ }^{8}$ Roberts WC. Congenital cardiovascular abnormalities silent until adulthood. In: Roberts WC, ed. Congenital heart disease in adults. Philadelphia: FA Davis, 1979:407-53.

9 Nanda NC, Gramiake R, Shah PM. Diagnosis of aortic root dissection by echocardiography. Circulation 1973;48:506-13.

${ }^{10}$ Cohen IS, Wharton TP jun. "Duplication" of aortic cusp: new M-mode echocardiographic sign of intimal tear in aortic dissection. Br Heart J 1982;47:173-6. 